
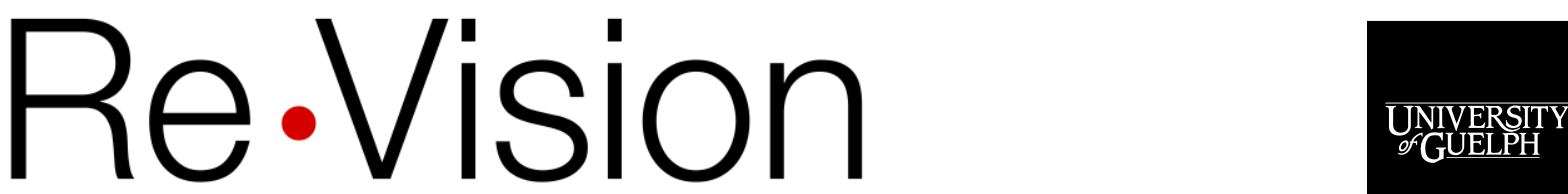

The Centre for Art and Social Justice

\title{
Recovering Uncertainty: Exploring Eating Disorder Recovery in Context
}

\author{
Andrea LaMarre \\ Massey University \\ Carla Rice \\ University of Guelph
}

This is a post-peer-review, pre-copyedit version of an article published in Culture, Medicine, and Psychiatry. The final authenticated version is available online at: https://doi.org/10.1007/s11013-020-09700-7

\section{Recommended citation:}

LaMarre, A., \& Rice, C. (2021). Recovering uncertainty: Exploring eating disorder recovery in context. Culture, Medicine, and Psychiatry. https://doi.org/10.1007/s11013-020-09700-7 


\section{Recovering Uncertainty: Exploring Eating Disorder Recovery in Context}

Andrea LaMarre ${ }^{1}$, Carla Rice

\section{Abstract}

Attending to the shades of grey in eating disorder recovery may help to illuminate possibilities for navigating recoveries in their full complexity and diversity. There is a need for more complexity and flexibility in understandings of the timelines, processes, endpoints, and versions of eating disorder recoveries. In this article, we explore eating disorder recovery as a dynamic, intercorporeal, and non-linear process. Drawing on interviews with 20 people doing significantly better than they were during a time of acute distress around food and body, we articulate "recoveries" in relation to four themes:

Fuzzy Logics of Time, Not Only Recovered, Recovery is Not All Sunshine and Rainbows, and The Life of Recovery. These themes speak to the ways in which participants struggled to articulate the temporalities of their recoveries, situated recovery as one among many events and processes that shaped their being in the world, resisted "too perfect" articulations of recovery journeys/ endpoints, and described preferred versions of and open-ended guidelines for recovery. We argue that eating disorder recoveries are as complicated and messy as lives themselves and are equally

\footnotetext{
${ }^{1}$ Research carried out while Andrea LaMarre was affiliated with the Department of Family Relations and Applied Nutrition, University of Guelph, Ontario, Canada. Parts of this manuscript have been drawn from Andrea LaMarre's doctoral dissertation, which is publicly available online through the University of Guelph Atrium.
} 
entangled in social contexts. We suggest that articulations of recovery be attuned to power dynamics as they operate in dictating which performances of eating disorders and recovery will be honoured as "legitimate" and whose pathways to recovery will be respected.

\section{Key words}

eating disorders, recovery, temporality, recovery model

Continued uncertainty about what "recovery" means—to clinicians, to people with lived experience, to supporters, and to the general public-is characteristic of the eating disorders field in 2020, despite efforts at reaching consensus or even provisional agreement on the subject (e.g. Bardone-Cone et al. 2010, 2018; de Vos et al., 2017). The voices of people living with or with a history of eating disorders are considered in articulations of recovery insofar as studies often quote people experiencing eating disorders and/or in recovery or capture aspects of recovery among those participating. Movement toward a patient-centered, recovery-model-oriented approach has begun, fueled by awareness of the need to look at individual and contextual understandings of the "end goal" of eating disorder treatment (e.g., Churruca et al., 2020; Dawson et al., 2014; Musolino et al., 2016).

However, a recovery model orientation remains largely tethered to eating disorders deemed severe and enduring (e.g., Calugi et al., 2017). While a recovery model may live in relation to medicalized discourses within treatment settings (Dawson et al., 2014; Musolino et al., 2016; Lester, 2019), the co-existence of such paradigms for 
assessing, and accessing, "recovery" sparks unspoken tensions within and beyond treatment spaces. People living with eating disorders are not always trusted to be "accurate" tellers of their stories; often, it is assumed that they are ruled by an "eating disorder voice," and linguistic framings such as "the anorexic" tend to subsume their multifaceted identities into an eating disorder (Holmes, 2016; Malson et al., 2004; Saukko, 2008). Ironically, these assumptions and orientations may limit people's capacity to envision recovery (Malson et al., 2011) and may set up adversarial treatment relationships (Boughtwood \& Halse, 2010). These relationships are also mediated by gendered, raced, classed, and abled assumptions that govern the boundaries of the who, what, and how of eating disorders-who these diagnoses are open to, who they exclude, and who is constructed as deserving of care. In exploring such dynamics, it becomes evident that eating disorders, care, and recovery are inextricably tied to power in and around treatment spaces (Lester, 2018, 2019). "Defining" or even describing recovery, thus, is not simply a sterile clinical or research exercise.

Societal power dynamics inform eating and weight-related prescriptions issued to those in society in general; in eating disorder treatment settings, prescriptions for eating and body management that run counter to broader perspectives can subject those in recovery to conflicting sets of instructions about health and wellness (LaMarre, Rice \& Bear, 2015; LaMarre \& Rice, 2016; Malson et al., 2011). Recovery may become a "performance" of meeting certain standards, which may exceed or differ significantly from population health guidelines (LaMarre \& Rice, 2017). Recovery may be reduced to eating and body management, which misses an opportunity to focus on quality of life 
issues that intersect with pathways to recovery, key components of the "what" of recovery (de Vos et al., 2017). While there is a near-constant refrain in the literature that "clinical reliance on biometrics (i.e., weight) and food intake, [is] insufficient, and does not address the psychological part of recovery" (Bardone-Cone et al., 2018, p. 78), how to consider the "psychological part" and what is considered to make up that "part" remains unclear. How this "psychological part" entangles with broader societal discourses and relationships, including those mentioned above, is still less clear.

In this article, we explore the messiness and meanings of recovery along with participants who have various experiences of diagnosis/non-diagnosis and treatment/non-treatment. We draw on interviews with 20 people who have experienced distress around food and in their bodies to explore possibilities for articulating recoveries in non-uniform, non-universalizing ways—without individualizing responsibility for its achievement. In this, we move away from a notion of recovery rooted in naïve empiricism - the idea that any credible description or demarcation of recovery can only emerge from empirical observation, that recovery itself is easily identifiable since it "simply mirrors external reality" (Teo, 2018, p. 133) —towards a layered heuristic that recognizes how embodied subjectivity, inter-relationality, temporality, and social context inform individuals' experiential understandings of recovery. The findings presented here respond to our research question: How do people who have experienced distress around food and body and their supporters understand the possibilities, processes, and outcomes of "eating disorder recovery" within a contemporary anglo-Canadian context? 


\section{Biopedagogies of Risk and Recovery}

Biopedagogies can offer a helpful theoretical frame for exploring how power circulates through recoveries and their expression. Biopedagogies are the subtle and diffuse messages that circulate everywhere in society and tell people what they need to do in order to be healthy, happy, and hard-working citizens (Wright \& Harwood, 2009). Perhaps the most obvious example of biopedagogies in the contemporary West is the uptake of anti-obesity discourses. Under such biopedagogies, fat bodies are routinely de-humanized and degraded (Saguy \& Ward, 2011; Rice, 2014, 2015). Morals are tied to bodies, and those inhabiting larger bodies learn they are unfit on a physical level and

on a moral one (e.g. De Brun, McCarthy, McKenzie \& McGloin, 2014; Rinaldi, Rice, Lind \& Kotow, 2020). Health is read into weight, with the assumption that we can ascertain from a glance whether we are measuring up to societal standards not just of appearance but also of productivity.

In relation to eating disorder recoveries, biopedagogies might be regarded as mapping the margins within which recoveries are made possible (or impossible). Assumptions about whose bodies fit-and are fit-serve to re-inscribe a constricted zone of "normality" into which all bodies should fit—neither "too fat" or "too thin" (Lupton, 2013). Such assumptions are also inflected with "healthism," wherein people are judged to be more or less morally valuable on the basis of their achievement of a particular version and vision of health (Crawford, 1980). Under this optic, people's bodies become "proof" of their attainment, or lack thereof, of idealized health. Practices assumed to result in "healthy bodies" are based in white, middle-class cultures, and include exercise and dietary behaviours that may be incongruent with the practices of other (sub)cultures 
(Brown-Bowers, Ward \& Cormier, 2017; Rice et al., 2020; Rinaldi et al., 2016).

Biopedagogies are implicated in the production and reproduction of surveillance over bodies that neither helps those struggling with distress in their bodies and around food nor makes it easy to "recover."

\section{Temporalities of Recovery}

In addition to potentially generating sets of instructions for being healthy that conflict with dominant health biopedagogies, mainstream recovery models prescribed in treatment settings are deeply enmeshed with normative discourses that dictate the space (e.g., formal therapeutic settings) and time (e.g. a linear pathway, perhaps with some steps "backward" but building toward wellness) in which recovery is expected to occur. In "managed care" settings common to Western medical systems (see Lester, 2018, 2019 for more on managed care), individuals seeking recovery enter into neoliberalized, resource-rationed systems; here, they are expected to be efficiently moved from illness to wellness and productivity. In these settings, their progress is measured externally using biometric markers such as "normal" eating and weight restoration (Gremillion, 2003; LaMarre et al., 2015; Lester, 2019).

Time in eating disorder treatment is dictated by varied—and competing—-models (Lester, 2019). One that aligns with broader configurations of time in Western cultures is neoliberal medicine's "managed care" time; this presupposes that recovering bodies can (if they work hard enough) conform to "normal" standards for bodies and health (Lester, 2019). A recovery-model oriented time is more open to difference, allowing for a "self" in time that is "misdirected" (Lester, 2019). It is possible that even an optic that frames selves as "misdirected" may implicitly assume a "proper" direction—however 
contextualized against the fabric of a person's life. Indeed, a recovery model potentially aligns with the individualizing march of neoliberal capitalism, configuring the subject as "in it on their own" even when they are surrounded by, impacting and impacted by, others in their relational configurations (Price-Robertson et al., 2017). The term "recovery," itself arguably connotes movement toward some category of experience deemed "normal" (Howell \& Voronka, 2012)—a socially constructed, and possibly unavailable or even undesirable place (Clare 2017; Davis, 1995).

Outside of the eating disorder literature, feminist theorist Allison Kafer (2013) proposes crip time; a "strange temporality" that reflects "a different way of being in the world, a creative, flexible, and non-linear way of relating to time" (Morrigan, 2017, p. 56). Articulating the flexibility, improvised quality, and entangled aspects of temporalities may be more in line with a "polyvalent," contextual, and multi-layered vision of recoveries (Pilgrim, 2009). Here, recoveries might be imagined as "site[s] of sociopolitical struggle over what lives are deemed liveable in the context of global neoliberalism" (McWade, 2015, p. 244). These socio-political struggles are also tied to differential access to "enabling resources" (Duff, 2012, p. 1389)—including people, material resources, and received strategies for coping - that constrain or open recovery. Recoveries, and hope for recoveries, are not achieved by individuals, but rather by collectivities (Bird et al., 2014; Price-Manderson et al., 2017) and maintained in a relational network, in time, and in space (Duff, 2016). A perspective on "recovery as both a personal and social experience" (Morrow \& Wiessler, 2012, p. 28) repositions recovery as deeply entwined with social inequities and possibilities (Morrow, 2013). We enter into our analysis with the perspective that it is important to consider recoveries in 
the contexts of people's lives, including their relationships with people and things in their worlds, their access to material, emotional, and other resources, and their timescales for/experiences of temporalities in recovery.

\section{The Language of Recovery}

Being given or taking an eating disorder label is identity work. Elsewhere, critical researchers have commented on how people negotiate their subjectivities in and/or outside of clinical and popular discourses on both eating disorders and recovery (e.g. Churruca, 2020; Holmes, 2018; Malson, 1998; Saukko, 2008; Shohet, 2007, 2018). For instance, Malson (1998) remarked on the "discursive struggle over interpretations of 'self' and 'anorexia'" (p. 144) in an analysis of the accounts of women diagnosed or selfdiagnosed with anorexia. In later work, Malson and colleagues $(2004,2011)$ continued to explore how the label of "anorexia" carries particularized expectations of subjectivity and selfhood that can limit self-expression outside of its boundaries. In analyzing treatment experiences of participants diagnosed with eating disorders, Eli (2014) noted the simultaneous relief and disappointment in gaining legitimacy via receiving a diagnostic label and engaging in treatment.

Many people fall outside of the boundaries of intelligibility through which eating disorders are interpreted, understood, and labelled. Black women and people of colour routinely face barriers to diagnosis and treatment (Cachelin et al., 2001; Gordon, 2006; Sinha \& Warfa, 2013; Thompson, 1994). Similarly, pervasive myths inhibit the recognition of eating disorders amongst LGBTQ+ individuals (Jones \& Malson, 2013; MacDonald, 2011; Rice et al. 2020), men (Collier, 2013), and people in larger bodies (Lebow, Sim \& Kransdorf, 2015; LaMarre, Rice \& Rinaldi, 2019). Those who have not 
had their eating disorders "legitimized" by the medical community may still articulate their experiences using diagnostic language but describe fraught and harmful relationships with medical professionals that at times have further entrenched their "disorders" (LaMarre, Rice \& Rinaldi, 2019).

Seeking and obtaining a diagnosis and engaging with the terminology of "eating disorder" carries significant weight for constructing the self. The label, language, and conceptualization of recovering/recovered similarly carries tensions for identity work. Conti (2018) frames recovery "as an identity journey," reflecting on the importance of prioritizing first-person perspectives on experiences that challenge commonplace versions of what recovery is or "should be." Churruca et al. (2020) similarly reflect on contradictions within which people in recovery may become caught that limit their ability to articulate themselves outside of clinical, medicalized terms. Shohet (2007) illustrates how people in recovery draw on clinical and popular articulations of recovery to articulate themselves, constructing "struggling to recover" and "full recovery" stories. In a close analysis of a woman's narratological work in eluding eating disorder diagnosis and treatment, Shohet (2018) goes on to describes how a participant distanced herself from clinical diagnosis and treatment by disengaging from narratives that gestured at disorder and a concordant need for recovery.

Musolino et al.'s (2016) work with those who have avoided eating disorder treatment brings into relief how seeking recovery as defined within a (narrow) clinical focus may conflict with people's own ideas of "self-care" (i.e. practicing restrictive eating). In later work focusing on anorexia deemed "severe and enduring," Musolino et al. (2020) explore how within cultural contexts that prize smallness and "control," 
embodied practices associated with anorexia can feel life safety. In this context, if recovery is seen as a rupture with these embodied practices, it is a request for people "to not only give up the protective and productive aspects of anorexia nervosa, but also to step outside of culture" (Musolino et al., 2020, p. 8).

What these and other studies reveal is the complexity of living in and outside of diagnostic and recovery categories; they suggest that discourses about eating disorders and recovery are not simply inscribed upon bodies but instead a part of the fabric people use to weave their identities. The ways in which researchers, treatment providers, and people in general discuss and perform "recovery" carry instructions that may conflict with biopedagogical instructions for health more broadly, delimiting the boundaries of recoveries into a narrow zone of intelligibility.

\section{Methods}

We describe above some tensions inherent in clinical and popular framings and use of the term "recovery"; accordingly, throughout this research we have been thoughtful about how we use the term in order to avoid foreclosing ideas about what it means to participants. As researchers, we have experienced our own relationships to the word recovery, which have shifted over time in relation to the various projects we have undertaken with people navigating complex and contradictory sets of instructions about how to be healthy. We, along with those interviewed, have quite literally "recovered" our lives from eating and body-related distress even though we, like our participants, still feel the pull of dominant dictates for eating and body management.

We thus did not require that participants had a diagnosis or a clinician-endorsed "recovery" to participate. We aimed to explore, together with participants, the discursive, 
material, and affective impacts of "recovery." As such, we invited co-definition and exploration of the term itself. Others have commented on the challenges associated with using the terminology of recovery while seeking to explore it (e.g. Conti, 2018). Further, as Levy, Wright \& Halse (2016) point out, researchers working in the health space we are to a certain extent bound up in the very discourses we seek to unpack (and the methods we use to unpack them), which inevitably shapes what we look for and what we find. In this project, the degree to which participants took up the invitation to define recovery varied, in part based on their experiences with formal/medical eating disorder services.

Working with participants, we negotiated meanings of recovery, for instance referring to "doing significantly better than during a period of acute distress." However, as noted, a part of the work with participants also involved explicitly engaging with the term "recovery" and what it has meant in their lives. As described in the results, interviews also surfaced for us and participants critiques of dominant ideas about "being well" or "wellness" and linear progress narratives that might be associated with "doing better" and/or the term "recovery." Resultantly, we have come to believe that there is value in holding onto the term as a means of describing the passage from intensive to quotidian struggle, while opening its terrain to contestation and critique.

Participants were recruited via the first author's social media accounts and list serve postings. Data collection took the shape of individual interviews conducted by the first author. After each interview, participants were invited to participate in a digital storytelling workshop to make a short film about their experiences, and asked if there was a person who supported them in their recovery they would like us to interview. The 
first author also interviewed 14 supporters and proceeded to make short films with 4 participants in recovery and one supporter. In this article, we report on interview data, though the insights we gained through deeper engagement with supporters and in making digital stories have shaped our analytic lens.

Participants were asked questions about their eating distress/eating disorder, and relationship to the term recovery, to treatment systems, and to others in their lives throughout the process. Participants' average age was 28 (range 19-41). Fourteen identified as heterosexual; the remaining six identified as pansexual, bisexual, or sexually fluid. Nineteen identified as women and one identified as gender nonconforming. Fifteen participants identified as white, one identified as Greek, and four as Asian. Participants described a range of diagnoses and symptoms, including binge eating, purging, restriction, over-exercise, and fixation on particular ways of eating. Participants were asked which terminology they preferred to refer to their eating/weight practices; the majority chose the language of "eating disorder," regardless of diagnostic status. Timelines for recovery were challenging for participants to articulate due to the entanglement of behaviours coded as "eating disordered," clinical encounters, relationships to recovery terminology, and other factors in their lives. Some discussed their eating disorders as recent (i.e., within the past year) whereas others described their disorders as being much further in their past (i.e., 15 years ago). In the extracts we share below, we have not offered demographic information alongside participant pseudonyms; this is a purposeful choice taken to minimize identifiability.

We conducted a thematic analysis (Braun \& Clarke, 2006) of the 20 interviews we describe above. The project received ethics approval from the University of Guelph 
ethics board REB\#16-12-277 We took up a critical feminist, new materialist theoretical lens to analyze patterns in the data at the latent level, seeking to understand themes in relation to broader social structures, discourses and physical objects (Braun \& Clarke, 2006). The first author coded the dataset and developed thematic areas organized around central ideas. Throughout data collection and analysis we aimed to engage in a "rethinking [of] the thinking that underpins the 'doing' of research practice" (Fullagar, 2017, p. 247-8). The first author engaged in reflexive praxis throughout by noting moments in which she felt attached to, challenged by, aligned with, and distant from the data. She discussed themes and reflections with the second author; together, the authors refined themes. This process of refining continued throughout write-up. We worked closely with theory in our analyses; rather than seeing the data as representing some singular external "truth," we explored the entanglement of participants' stories, our own stories, and the array of theories we use to make sense of experiences. We use theory to think about subjectivity as embodied (i.e., expressed through non-verbal cues, felt in fleshy ways by researcher and participant alike) as well as being ideological (i.e., tied to power discourses typically configured as "external to" subjects) (Chadwick, 2017).

A summary of themes identified was shared with participants; several responded that they found the work to be representative of their experiences and that they enjoyed participating in the research. This manuscript is one of several articles stemming from the research; themes on which we report here represent central parts of meaning the researchers made of participant stories about recovery. 
We make no claims to neutrality and acknowledge that our own embodied subjectivities and histories impact and are impacted by the research we do. We are both white, cis-gender women. AL is young, heterosexual, and married; she passes easily as able-bodied, and identifies as having a history of an eating disorder, though she continues to trouble the categories of illness and wellness as her "recovery" progresses. She is embedded in research, advocacy, and lived experience communities which likely facilitated her engagement with prospective participants; this could be framed as either a strength or a limitation, but certainly shaped at least some of the "results" we present here. CR identifies as queer, middle-aged, white working class, and coupled; as a woman with a history of significant food and weight distress; and as someone who, having worked in and around eating disorder treatment programs, decided to become an academic as a way of breaking the stranglehold of hyper-individualizing, pathologizing discourses over these spaces. Elsewhere, we continue to write about our embodied engagement with the "data" in this project; within the space limitations of this article, we note these spaces of belonging to foreground the "stakes" we have in this analysis and the lenses and embodiments we brought to the work.

\section{Analyses \\ Recovery is Life}

The various facets of our analysis contribute to an overall picture of how recovery might be conceptualized as life itself-not in a way that presumes that it is the central focus but acknowledging that there is no singular way of understanding what recovery is or could be for different people. Together, participants' experiences build to conceptualize a vision of recovery that is grounded in lived realities-including 
embodied, contextual ways of being and temporal structures that exceed a linear narrative that positions people as "before" and "after" and that resists a singular destination point. Participants' stories challenged an assumption of linearity in recovery. Unsatisfied with prior descriptions of recovery and how to achieve it offered by healthcare providers, various publics, family members, friends, and others in their lives, participants called for an embodied and embedded version of recovery. For many, recovery was not the defining aspect of their being, but one facet among several. We describe four themes below, centered around four core tensions we noted in participants' accounts.

\section{Fuzzy Logics of Time.}

The first core tension we noted in participants' accounts related to how participants felt compelled to construct timelines for their recoveries, and yet often struggled to do so and did not align with a linear timeline model. Many participants described their recovery timelines as fuzzy or foggy. Deb, for example, had trouble articulating her recovery trajectory due to her "inability" to recall large segments of her experience:

The scary thing is, I don't even remember a whole lot of the last 10 years. I remember a little, sort of bits and pieces, and sometimes I don't even remember which can first. (Deb)

Lester (2019) comments on how eating disorders "make" and "unmake" time, "fragment[ing] experiences of self and scatter[ing] attempts at concentrated action" ( $p$. 144). Given that in treatment, people may be expected to construct a "timeline" of their experiences that bounds the eating disorder's presence and role in one's life (Lester, 
2019), it is perhaps unsurprising that the inability to construct a timeline might be considered a "scary thing," in the way that Deb describes.

Similarly, Sophie's account attests to the challenges of articulating points-in-time associated with her eating disorder and recovery:

$\ldots$ with the onset of my eating disorder, there was no event or anything that I can point at and say "okay, it all started there, at that time." [...] a few things just merged together and made it start, so it's the same thing that happened with the end. At some point I just realized that $[\ldots]$ I think I'm eating kind of normally now. (Sophie)

Participants' timelines of recovery did not always suggest a kind of illuminating event that made them realize either the onset or the remission of their eating disorders. Instead, as Sophie articulated, "a few things just melded together" situating the experience of recovery in the rest of her life. Elsewhere, a "turning point" has been considered key to recovery experiences (e.g. Matusek \& Knudson, 2009; Moulding, 2016; Stockford et al., 2019) and described as a feature present within narratives of those aligning with a "full recovery" orientation (Shohet, 2007). Many of our participants' narratives resisted this structure, aligning more with the kind of fragmented temporality that has been associated with eating disorders themselves (Lester, 2019).

Some participants relied on supporters to help them piece together their recovery trajectories, and to remember what they had been like during the time of their eating disorders. Participants accomplished this articulation in part by assembling a timeline in concert with loved ones. For example, reflecting on discussions with her fiancé about 
her illness and recovery, Isabel noted how memories entwine to generate her overall picture of her pathway.

He remembers things very vividly and I don't have as strong of a memory from that time about things I did, or what I looked like, or how I acted. He'll bring it up from time to time about how, how far we've come and what the experience was like for him and us_-and then, I think I tend to bury it a lot because, and I don't know if this is common, but I almost have like flashback-style memories of things that'II happen just randomly [...] and I barely remember being there but I can remember it very vividly, certain things. (Isabel)

Isabel's account reveals how both the eating disorder and the recovery process can bring the past into the present in relational and/or intrusive ways. In the extract, Isabel describes two different ways of encountering time; in her relational articulation, the past becomes intelligible through co-constructed memories with her partner. Her "flashback-style" memories, on the other hand, may be shorter, more visceral, and particularized. While offering no information about the content of the memories that "surface" or are brought into being in either way, Isabel comments on how the collectively-generated or relational memories are more of an overall picture of the past brought into comparison with the present, whereas the intrusive memories are of "certain things," suggesting particular moments rather than an overall sense of what it was to have "been there."

Not Only Recovered.

Participants did not always consider their eating disorders or recoveries to have been the single most important part of their lives, and many troubled the use of the term 
"recovery". A core tension developed between seeing the eating disorder and recovery as formative events or pieces of identity and also wanting distance from these experiences. For example, Alina configured her eating disorder as one of several negative life events from her teenage years. Asked about how she would refer to her experience after acute distress around food, weight, and shape, she noted:

I would never call it recovery, I feel like I don't want to like dramatize it too much... it's just one of many dumb things that I did. (Alina)

By framing the eating disorder as a "dumb thing" that she "did," this extract arguably reinforces a potentially problematic framing of eating disorders as a choice. However, we might consider this rhetorical distancing from the idea of what a "dramatic" eating disorder might be or look like, or what kind of clinical intervention might be required to achieve recovery. This distancing is reminiscent of critical feminist work on eating disorders wherein participants construct themselves as other than eating disordered in relation to stereotypical portrayals of vanity and choice (e.g. Malson, 1998). Here, Alina distances herself from constructions of eating disorder and recovery - and yet, through her self-selection into the study, we might infer at least some self-construction in relation to moving through eating distress. For Alina, this moving through was tied to moving forward in other areas of her life, rather than pursuing "traditional" forms of treatment. For her, and some other participants, "moving forward" meant focusing more on other aspects of life.

Reluctance to identify with recovery terminology might be understood as a form of resistance to clinical discourses that interpret eating disorders as pathologies that completely overpower individuals' psyches and lives. This desire for distance also 
brought tensions to the fore for those who did feel that the eating disorder had significantly impacted their subjectivity, but also wanted to move beyond identification with the eating disorder and even an identity as "someone who has recovered." As Elizabeth noted:

A big thing for me is not making it my one and only. Because then I feel like I want to hold onto it more, and I feel like it's more a part of my identity. But I really don't want it to be a part of my identity. But a part of me does. [...] I'm tired of letting things get in the way of what I want to do. And I'm just not willing to let them anymore, so if someone has a problem, like whatever. I have goals that are bigger than you, so I'm gonna pursue those. (Elizabeth)

In order to, as she also phrased it, "get on with her life," the eating disorder and recovery needed to become less of a focus. Elizabeth expanded on the need to move beyond the eating disorder/ recovery identity as core to her being by reflecting on her larger life goals and how those interacted with the disorder. Recovery was thus enabled by focusing on other aspects of life.

\section{Recovery is Not All Sunshine and Rainbows.}

Another core tension participants articulated was related to not aligning with a linear trajectory for recovery while simultaneously desiring a "roadmap" for recovery that would have better prepared them for the experience. They hoped for a roadmap would articulate recoveries in a way that reflected the experiential complexity of recovering into a world that excludes many bodies. Some participants expressed how they had desired to enact a "perfect" recovery, which paradoxically contributed to delays in seeking help during relapse, as Emily describes: 
I went from eating disorder to "I'll have a perfect recovery" and so I followed the meal plan. I wasn't against it, I've always loved food, so I enjoyed all the different variety I was then allowed, and the different desserts, and the snack. The only thing I didn't like was the Ensure that I had to have and I didn't like that I couldn't be active $[\ldots]$ I went for my final year of high school, kind of seeing the medical doctor out of the (hospital) program, and we never addressed the underlying psychological issues, I think, and that was my own surface-level treatment of everything. Like "everything's fine, like I'm happy." (Emily)

In this extract, we can see how expectations for perfection in recovery hindered a deeper sense "address[ing] the underlying psychological issues". Emily refers to the version of recovery she enacted as "surface level," suggesting that engagement with this perfection did not enable a deeper, felt sense of "being well."

The unattainable perfection of representations of recovery was also associated, for some, with an imperative to "love your body." Such an imperative was highly gendered, and imposed standards that participants noted they felt they needed to live up to in order to perform fit femininity and demonstrate that they had achieved recovery. As Carrie reflected:

The Dove commercials, that are like "all women should feel beautiful all the time!" and, and I'm like "that's fucking bullshit, why don't we just all feel like ourselves, and feel human, and feel like smart, or caring", like they shifted the focus from "okay, so you, most women should feel ugly" to "women should feel beautiful." But they're still making us fixate on how we look as the most important thing about ourselves. [...] And if you don't feel beautiful, then you suck as a human 
being. You're supposed to live in this world and always feel beautiful in the skin you're in, no matter what you look like, and if you don't, then again, the problem is with you. (Carrie)

Carrie reveals that the issue with this discourse is not necessarily it's positivity, but rather who was made responsible in the love your body discourse-the individual ("if you don't [...] the problem is with you"). In this extract we can also see how Carrie refers to an expectation to continue to be focused on the body—but to make a move from body hatred to body love. This discourse of body love is a part of a sociocultural surround that is rich with conflicting imperatives for body management that make navigating recovery fraught.

Many participants articulated recovery as other-than-perfect; indeed, some engaged directly with the idea of letting oneself be imperfect. Sherice shared:

I think for me right now my biggest thing that I'm working on is being compassionate towards myself, and for me that means letting myself not be perfect and everything doesn't have to be done in a certain way. (Sherice) Exploring recovery beyond perfection required participants to let go of a dominant vision of the perfect image of recovery-which is not easily done, particularly when so few visions of recovery are available to those seeking them.

The Life of Recovery.

A final tension we explore in participants' perspectives on recovery relates to their seeking out of representations on recovery with which they could resonate, but their configuring of their own recoveries as unique. For instance, Julia now locates 
herself as her own best role model for recovery as she recalls past understandings of whether it was possible to live without an eating disorder:

[I]n a lot of ways, I was almost like resigned to just living with it for the rest of my life, which is really sad, but it just goes to show how bad it was. I wish I could almost go back and give a glimpse of what life was like now, like it's not by any means sunshine and rainbows, it's not a million times better, but it is a life, you know? (Julia)

Julia's vision of recovery entails "going back" and speaking to herself about what recovery would be like; in thinking through recovery and who might be a credible source for inspiration, Julia configures herself as her best prospective role model. Others similarly endorsed carving out their own recovery pathways; Ellen, for instance, noted that her version of recovery was "just something I made up on my own." Caitlin similarly remarked that she acted as her "own role model" in recovery:

I was kind of my own role model and I, because I had such a great, a strong family. It was great to have that constant support and those constant role models behind me the whole time as well. (Caitlin)

She specified, here, that any other role models she looked to were not those who had experienced eating disorders, but rather family members who lived relatively unencumbered by significant distress around food, weight, and shape. Others echoed similar sentiments, insisting that recovery for them be evaluated in relation to their own experiences and embodied spaces of belonging. 
I think it's that idea of everyone's eating disorder is a little bit different. And the importance of listening to the individual. And I think asking the individual what they see as recovered, what recovery looks like to them. (Emily)

Regardless of the actual content of their statements about what recovery "is," all expressed that a critically important part of getting beyond distress was the presence of others who listened to them, believed their versions of recovery and bought into their goals, within and outside of recovery—as illustrated in the extract from Emily, above.

\section{Discussion}

Participants' narratives encapsulated the complex interweaving of time, relationality, and space as they enacted and performed their recoveries in a matrix of biopedagogical instructions about bodily behaviours. Participants described the constraints of measuring their recoveries against articulated or imagined "perfect" standards for recovery—engaging in the right recovery practices, attaining the right body, achieving the right end goals and accomplishing these within the right timelines. As Allison, Warin \& Bastiampillai (2014) note, "young people (and young women in particular) learn from an early age that their bodies require constant surveillance, modification and improvement" (p. 116) particularly in relation to food and exercise practices. For participants, recovery discourses formed at least one part of this surveillance apparatus, calling into question their ability to claim "recovery" as aspect of identity.

Ongoing self-surveillance has been a feature of recovery models beyond the eating disorder context in their emphasis on people's need to manage themselves and their bodies such that they reduce the risk of relapse (Price-Robertson et al., 2007). 
This self-surveillance dovetails with population-based biopedagogies encouraging constant self-management around food and bodily practices (Wright \& Harwood, 2009) and discourses of discipline and management of feminine bodies in particular (Gill, 2007; Rice, 2014). Together, these can create a constrained definition of recovery closed to those who do not perform recovery as "expected." In describing how they felt pressure to "live up" to some externally imposed strictures for what and how a body in recovery might be, participants' responses raise the question of whether striving for a "perfect" or prescriptive notion of recovery might actually work to the detriment of a true sense of being well, at least for some.

Given that recovery in context is "a site of socio-political struggle over what lives are deemed liveable in the context of global neo-liberalism" (McWade, 2015, p. 244), we might consider how discourses of "choosing recovery" allow the biomedical psychiatric complex to co-opt liberal individualism to promote an version of recovery not available to all (Howell \& Voronka, 2012; Morrow, 2013; Poole, 2011). This "choice" based framing of recovery ignores the complex and ongoing affective and relational aspects of eating disorders and their care (Lester, 2018, 2019). Power and privilege, representation, and temporality inform recovery pathways in ways that complicate a simple choice-to-be-whole narrative so commonly associated with eating disorder recovery. Participants' accounts raise the possibility of carving out a space for recoveries to not be about returning to an ostensible "before" or reaching a place free of problems. Given the ways in which the perspective of returning to a state "before" may rub up against a more situated, specific version of reclaiming identity for those experiencing eating disorders, it may be more apt to use a term such as "reclamation" 
(Conti, 2018). Reclamation "denotes the recovery of that which was harmed or hurt (a recognition that something has been taken); a return, or more provocatively still, a turn to a generative, lively and vital state of being" (Friedman, Rice \& Lind, 2020, p. 8).

Including participants who had not been diagnosed might be framed as a limitation of this study, as might following a semi-structured interview guide that introduced significant variability into how participants told their stories. Further, our sample size and Canadian focus limit the generalizability of the work. However, our open recruitment criteria arguably allowed us to engage with participants who were, at times, unsure of what or where they "were." Some participants were occasionally engaging in symptoms that would be coded as eating disordered; others questioned weight thresholds that had been suggested for them by professionals. Some had not received treatment for their disorders and had recovered with the help of more informal supports. In these cases, participants did not engage "as expected" with the medicalpsychiatric complex, and thus had no "proof" for their recovery. Others sought proof for their recovery in popular depictions of recovery and found that they did not cleanly fit into the before or after (see also Conti, 2018), again speaking to experiences of liminality for those neither "normal" nor "pathological." Recognizing their "abnormality" around food, but not diagnosed along medical lines, they became liminal in their expression of "abnormal abnormal" (McClintock, 2001; i.e., not fitting the particular codified set of behaviours that have been labelled pathological) behaviours around food, weight, and shape. Their behaviours significantly impacted their lives, but were not deemed "medically significant" to the extent that they might receive a diagnosis, which carries a certain seal of legitimacy (Eli, 2014). 
In recovery, participants may also become trapped in between normal and abnormal, a state mediated by others' perspectives on both their corporeal presentations and their behaviours around food in relation to social norms. This is compounded by a significantly lacking consensus on what recovery means (BardoneCone et al., 2010) that can result in rather participants' subordination to the particular perspectives on recovery espoused by clinicians, loved ones, and people in the general public—which may or may not conflict (Holmes, 2018; Noordenbos, 2011a,b). On the other hand, the consensus itself may operate as another kind of regulatory ideal. Participants' self-questioning itself reveals the tenacity of clinical discourses around eating disorders for delineating illness, wellness, and legitimacy.

\section{Implications}

People with eating disorders do not recover without knowledge of what recovery is "supposed" to be (Conti, 2018; Holmes, 2018). Indeed, they may articulate their subjectivities in relation to clinical and other discourses about eating disorders, recovery, and health in ways that materially impact their engagement-or nonengagement—with eating disorder treatment (Shohet, 2007, 2018). Participants in our study were cognizant of stereotypes around eating disorders, and of doctors', family and friends' reactions to their "imperfect" recoveries. These all informed their orientations to "recovery" as a concept, and the degree to which this featured in their lives. Aligning with earlier work on eating disorders and recovery that illustrates the importance of not discounting the agency of those experiencing distress in their bodies and around food (e.g. Malson et al., 2011; Musolino et al., 2016, 2020; Saukko, 2008) this finding further 
illustrates the importance of attending to the situated experiences of those seeking "recovery."

Participants' recoveries, though they were linked in some ways, were as diverse as lives themselves. Differences and similarities were tethered to participants' cultures, experiences of family and support, varied embodiments, orientations to the dominant social status quo and living in similar geographic locations. While our sample was relatively small and homogeneous, this holds importance for considering how recovery is narrowly represented as something only open to thin, young, white women (LaMarre \& Rice, 2017)—much like eating disorders themselves (Thompson, 1994; Nasser \& Malson, 2009). Participants' accounts speak to the need to avoid mandates around what recoveries must look like-accounts that frequently accompany attestations of recovery in research and clinical settings. The concept of a turning point, and the ways in which participants' stories contested the idea of a turning point being necessary or even possible to articulate, might provide a useful example. In a systematic review and meta-synthesis of AN recovery studies, Stockford et al. (2019) suggest that "to move towards recovery the individual must reach a 'turning point' where they develop insight into the function and consequences of $\mathrm{AN}$ and commit and take responsibility for recovery" ( $p, 343)$. However, with awareness of the ways in which such dictates become a part of the material people use to construct recovered subjectivities (Shohet, 2007,2018 ), we wonder about what the impact of these messages might be for those working through recoveries. While a turning point may well hold significant meaning for individuals navigating eating disorder recovery, it is important to consider which stories 
of recovery have been analyzed when compiling definitions of the concept-and whose recoveries might be left out of this picture.

Participants' multifaceted and varied accounts of recovery, their degree of engagement with dominant discourses on recovery, and their varied trajectories to recovery illustrate the dynamic, interactional, and intercorporeal nature of recovery, as well as the intertwined nature of recovery discourses with dominant conceptualizations of time, developmental trajectories, and normative performances of selfhood. Saying that recovery is life is more than a catchy way of framing this multifaceted construct, it is a way of acknowledging that we may never alight on a commonly-shared definition of recovery, but this does not need to be problematic. Lester (2019) points out how existing treatment systems for eating disorders standardize metrics for care in ways that limit a full embodiment of a recovery model. Evidently, system-level change is required to honour individual needs and definitions of recovery. Such a system would be undergirded by a fundamental respect for difference and a more consistent commitment to avoiding the pitfall of subsuming the identities of those with eating disorders and in eating disorder recovery into the eating disorder itself (Malson et al., 2004, 2011; Saukko, 2008). It would provide better access not only to any kind of care, but to the kind of care that might allow those in distress to determine ways of moving through distress as it relates to their own experience as opposed to presuming that all people with distress around food, weight, and shape require the same approach in order to recover.

This approach to recovery may sound utopic, but the ask emerging from participants' accounts is not outrageous or beyond the scope of reasonable, and 
meaningful, practice. Fundamentally, it is an ask for honouring lived experience. At the heart of enacting such an approach, however, is a reconsidering of power dynamics as they operate in dictating which performances of eating disorders and recovery will be honoured as "legitimate" and whose pathways to recovery will be respected.

\section{Acknowledgments}

We wish to acknowledge and thank our participants for their engagement with the research. We also acknowledge the scholars and activists in the eating disorders field who we have learned from and whose efforts have paved the way for social justiceoriented and feminist research.

\section{Funding}

This study was funded by the Canadian Institutes of Health Research (grant number: Vanier Scholar Doctoral Award 2014-2017) and the Ontario Ministry of Health and Long-Term Care (Ontario Women's Health Scholar Award 2017-2018). AL's dissertation research was generously funded by the Vanier Canada Doctoral Scholarship (ClHR) and subsequently by the Ontario Ministry of Long-Term Care through the Ontario Women's Health. The views expressed in the material are ours and do not necessarily reflect those of the Ministry of Health and Long-Term Care.

\section{Compliance with Ethical Standards}

\section{Conflict of interest:}

On behalf of all authors, the corresponding author states that there is no conflict of interest. Funding for this study is noted in the point directly above. 


\section{Ethical Approval}

All procedures performed in studies involving human participants were in accordance with the ethical standards of the University of Guelph ethics board and the Canadian Tri-Council Policy Statement: Ethical Conduct for Research Involving Humans and with the 1964 Helsinki declaration and its later amendments or comparable ethical standards.

\section{References}

Ålgars, Monica, Markku Anttonen and Jaana Suokas 2018. "Developing an outpatient unit for patients with severe and enduring anorexia nervosa."

Allison, Stephen, Megan Warin and Tarun Bastiampillai 2014. "Anorexia nervosa and social contagion: Clinical implications." Australian \& New Zealand Journal of Psychiatry 48(2): 116-120.

Bardone-Cone, Anna, Megan B. Harney, Christine R. Maldonado, Melissa A. Lawson, D. Paul Robinson, Rona Smith and Aneesh Tosh 2010. "Defining recovery from an eating disorder: Conceptualization, validation, and examination of psychosocial functioning and psychiatric comorbidity." Behavioural Research and Therapy 48(3): 194-202.

Bardone-Cone Anna M., Rowan A. Hunt and Hunna J. Watson 2018. "An overview of conceptualizations of eating disorder recovery, recent findings, and future directions." Current Psychiatry Reports 20, Art. 79.

Bird, Victoria, Mary Leamy, Jerry Tew, Clair Le Bouteillier, Julie Williams and Mike Slade 2014. "Fit for purpose? Validation of a conceptual framework for personal 
recovery with current mental health consumers." Australian and New Zealand Journal of Psychiatry 48(7): 644-653.

Boughtwood, Desiree and Christine Halse 2010. "Other than obedient: Girls' constructions of doctors and treatment regimes for anorexia nervosa." Journal of Community and Applied Social Psychology 20(2), 83-94.

Braun, Virginia and Victoria Clarke 2006. "Using thematic analysis in psychology." Qualitative Research in Psychology 3(2): 77-101.

Brown-Bowers, Amy, Ashley K. Ward and Nicole Cormier 2017. "Treating the binge or the (fat) body? Representations of fatness in a gold standard psychological treatment manual for binge eating disorder." Health 21(1): 21-27.

Cachelin, Fary M., Ramona Rebeck, Catherine Veisel \& Ruth H. Striegel-Moore 2001. "Barriers to treatment for eating disorders among ethnically diverse women." International Journal of Eating Disorders 30(3): 269-278.

Chadwick, Rachelle 2017. "Embodied methodologies: Challenges, reflections and strategies". Qualitative Research 17(1): 54-74.

Churruca, Kate, Jane M. Ussher, Janette Perz and Frances Rapport 2020. “It's always about the eating disorder': Finding the person through recovery-oriented practice for bulimia." Culture, Medicine, and Psychiatry 44(2): 286-303.

Clare, Eli 2017. Brilliant imperfection: Grappling with cure. Durham, NC: Duke University Press.

Collier, Roger 2013. "Treatment challenges for men with eating disorders." Canadian Medical Association Journal 185(3): E137-E138. 
Conti, Janet 2018. "Recovering identity from anorexia nervosa: Women's constructions of their experiences of recovery from anorexia nervosa over 10 years." Journal of Constructivist Psychology 31(1): 72-94.

Crawford, Robert 1980. "Healthism and the medicalisation of everyday life." International Journal of Human Services 10(3): 365-388.

Dawson, Lisa, Paul Rhodes \& Stephen Touyz 2014. "The recovery model and anorexia nervosa." Australian \& New Zealand Journal of Psychiatry 48(11): 1009-1016.

Davis, Lennard J. 1995. Enforcing normalcy: Disability, deafness, and the body. New York: Verso.

De Brun, Aoife, Mary McCarthy, Kenneth McKenzie \& Aileen McGloin 2014. "Weight stigma and narrative resistance evident in online discussions of obesity." Appetite 72(1): 73-81.

de Vos, Jan Alexander, Andrea LaMarre, Mirjam Randstaak, Charlotte Ariane Bijkerk, Ernst T. Bohlmeijer \& Gerben J. Westerhof 2017. "Identifying fundamental criteria for eating disorder recovery: a systematic review and qualitative meta-analysis." Journal of Eating Disorders 5(34)

Duff, Cameron 2012. "Exploring the role of 'Enabling Places' in promoting recovery from mental illness: A qualitative test of a relational model." Health and Place 18(6): $1388-1395$.

Duff, Cameron 2016. "Atmospheres of recovery: Assemblages of health." Environment and Planning 48(1): 58-74.

Eli, Karin 2014. "Between difference and belonging: Configuring self and other in inpatient treatment for eating disorders." PLoS One 9(9): e105452. 
Fox, Nick J. \& Alldred, Pam 2015. "New materialist social inquiry: designs, methods and the research-assemblage." International Journal of Social Research Methodology 18(4): 399-414.

Friedman, May, Rice, Carla \& Lind, Emma (accepted). "A high-risk body for whom? On fat, risk, recognition and reclamation in restorying reproductive care through digital storytelling." Feminist Encounters: A Journal of Critical Studies in Culture and Politics

Fullagar, Simone 2017. "Post-qualitative inquiry and the new materialist turn:

Implications for sport, health and physical culture research." Qualitative Research in Sport, Exercise and Health 9(2): 247-257.

Gill, Rosalind 2007. "Postfeminist media culture: Elements of a sensibility." European Journal of Cultural Studies 10(2): 147-166.

Gordon, K.H., Brattole, M.M., Wingate, L.R. \& Joiner, T.E. (2006). "The impact of client race on clinician detection of eating disorders." Behavioural Therapy, 37(4), 319325.

Gremillion, Helen (2008). "The race and class politics of anorexia nervosa: Unravelling white, middle-class standards in representations of eating problems." Pp. 218238 In Moss, Pamela \& Katherine Teghtsoonan. (Eds) Contesting illness: Processes and practices. Toronto: University of Toronto Press.

Hay, Phillipa J., Stephen Touyz \& Rishi Sud 2012. "Treatment for severe and enduring anorexia nervosa: A review." Australia and New Zealand Journal of Psychiatry 46(12): 1136-44. 
Holmes, Su 2018. “(Un)twisted: Talking back to media representations of eating disorders." Journal of Gender Studies 27(2): 149-164.

Holmes, Su 2016. "Between feminism and anorexia: An autoethnography." International Journal of Cultural Studies 19(2): 193-207.

Howell, Alison \& Jijian Voronka 2012. "Introduction: The politics of resilience and recovery in mental health care." Studies in Social Justice 6(1): 1-7.

Jones, Rebecca \& Helen Malson 2013. "A critical exploration of lesbian perspectives on eating disorders." Psychology and Sexuality, 4(1): 1-27.

Kafer, Alison 2013. Feminist queer crip. Bloomington, IN: Indiana University Press.

LaMarre, Andrea, Carla Rice \& Merryl Bear 2015. "Unrecoverable? Prescriptions and possibilities for eating disorder recovery." In Khanlou, Nazilla \& F. Beryl

Pilkington. Women's mental health: International perspectives on resistance and resilience in community and society (pp. 145-160). Toronto: Springer Press.

LaMarre, Andrea \& Carla Rice. Hashtag Recovery: \#Eating Disorder Recovery on Instagram. Social Sciences 6(3):68.

LaMarre, Andrea, Jen Rinaldi \& Carla Rice 2019. "Tracing fatness through the eating disorder assemblage." In Rinaldi, Jen, Carla Rice and May Friedman, M., (Eds.) Thickening Fat: Fat Studies, Intersectionality and Social Justice. New York: Routledge.

Lamoureux, Mary Mei Ha \& Joan L. Bottorff 2005. "“Becoming the real me”: Recovering from anorexia nervosa." Health Care for Women International, 26(2): 170-188. 
Lebow, Jocelyn, Leslie A. Sim \& Lisa N. Kransdorf 2015. "Prevalence of a history of overweight and obesity in adolescents with restrictive eating disorders." Journal of Adolescent Health 56: 19-24.

Lester, Rebecca J. 2018. "Ground zero: Ontology, recognition, and the elusiveness of care in American eating disorders treatment." Transcultural Psychiatry 55(4): 516-533.

Lester, Rebecca 2019. Famished: Eating disorders and failed care in America. Berkeley, CA: University of California Press.

Levy, Gary, Christine Halse and Jan Wright 2016. "Down the methodological rabbit hole: Thinking diffractively with resistant data." Qualitative Research 16(2): 183197.

Lupton, Deborah 2013. Fat. New York, NY: Routledge.

MacDonald, Danielle E. 2011. "Impossible bodies, invisible battles: Feminist perspectives on the psychological research on and treatment of eating disorders in queer women." Journal of Gay \& Lesbian Social Services 23(4): 452-464.

Malson, Helen, Lin Bailey, Simon D. Clarke, Janet Treasure, Gail Anderson \& Michael Kohn 2011. 'Un/imaginable future selves: A discourse analysis of in-patients' talk about recovery from an "eating disorder." European Eating Disorders Review 19(1): 25-36.

Malson, Helen, Mark Finn, Janet L. Treasure, Simon D. Clarke \& G. Anderson 2004. "Constructing 'the eating disordered patient': A discourse analysis of accounts of treatment experiences." Journal of Community \& Applied Social Psychology 14(6): 473-489. 
Malson, Helen 1998. The thin woman: Feminism, post-structuralism and the social psychology of anorexia nervosa. New York, NY: Routledge.

Matusek, Jill Anne and Roger M. Knudson 2009. "Rethinking recovery from eating disorders: Spiritual and political dimensions." Qualitative Health Research 19(5): 697-707.

McClintock, Anne 2001. Double crossings: Madness, sexuality and imperialism. Vancouver: Ronsdale Press.

McWade, Brigit 2015. "Temporalities of mental health recovery." Subjectivity, 8: 243260.

Morrigan, Clementine 2017. "Trauma time: The queer temporalities of the traumatized mind." Somatechnics 7(1): 50-58.

Morrow, Marine \& Julia Weisser 2012. "Towards a social justice framework of mental health recovery." Studies for Social Justice 6(1): 27-43.

Morrow, Marina 2013. "Recovery: Progressive paradigm or neoliberal smokescreen?" In LeFrancois, Brenda A., Menzies, Robert \& Reaume, Geoffrey (Eds.) Mad Matters: A Critical Reader in Canadian Mad Studies (pp. 323-333). Toronto, ON: Canadian Scholar's Press.

Moulding, Nicole T. 2016. "Gendered intersubjectivities in narratives of recovery from an eating disorder." Affilia: Journal of Women and Social Work 31(1): 70-83.

Musolino, Connie, Megan Warin, Tracey Wade \& Peter Gilchrist 2016. "Developing shared understandings of recovery and care: a qualitative study of women with eating disorders who resist therapeutic care.” Journal of Eating Disorders 4(36) 
Musolino, Connie Marguerite, Megan Warin \& Peter Gilchrist 2020. "Embodiment as a paradigm for understanding and treating SE-AN: Locating the self in culture." Frontiers in Psychiatry 11(534)

Noordenbos, Greta 2011a. "Which criteria for recovery are relevant according to eating disorder patients and therapists?" Eating Disorders 19(5): 441-451.

Noordenbos Greta 2011b. "When have eating disordered patients recovered and what do the DSM-IV criteria tell about recovery?" Eating Disorders 19(3): 234-245.

Pilgrim, David 2009. "Recovery from mental health problems: Scratching the surface without ethnography." Journal of Social Work Practice 23(4): 475-487.

Price-Robertson, Rhys, Lenore Manderson \& Cameron Duff 2017. "Mental ill health, recovery and the family assemblage." Cultural Medicine and Psychiatry 41(3): 407-430.

Rice, Carla 2014. Becoming women: The embodied self in image culture. Toronto, ON: University of Toronto Press.

Rice, Carla 2015. "Re-thinking fat: From bio- to body becoming pedagogies." Cultural Studies <=> Critical Methodologies (Special Issue on Biopedagogies and/of Public Health) 15(6): 387-397.

Rice, Carla, Karleen Pendleton Jiménez, Elisabeth Harrison, Margaret Robinson, Jen Rinaldi, Andrea LaMarre, \& Jill Andrew Forthcoming 2020. "Bodies at the intersection: Reconfiguring intersectionality through queer women's complex embodiments." Signs: Journal of Women in Culture and Society 40(1): 1-24. 
Rinaldi, Jen, Carla Rice, Emma Lind \& Crystal Kotow 2020. "Mapping the circulation of fat hatred." Fat Studies: An Interdisciplinary Journal of Body Weight and Society, 9 (1): $37-50$.

Rinaldi, Jen, Carla Rice, Andrea LaMarre, Karleen Pendleton Jiménez, Elisabeth Harrison, May Friedman, Deborah McPhail, Margaret Robinson \& Tracy Tidgwell 2016. “'Through Thick and Thin”: Storying queer women's experiences of taking up and resisting idealized body images and expected body management practices." Psychology of Sexualities Review (PoSR) 7(2): 63-77.

Saguy, Abigail C. \& Anna Ward 2011. "Coming out as fat: Rethinking stigma." Social Psychology Quarterly 74(1): 53-75.

Saukko, Paula 2008. The anorexic self: A personal, political analysis of a diagnostic discourse. New York, NY: SUNY Press.

Shohet, Merav 2018. Beyond the clinic? Eluding a medical diagnosis of anorexia through narrative. Transcultural Psychiatry, 55(4): 495-515.

Shohet, Merav 2007. Narrating anorexia: "full" and "struggling" genres of recovery. Ethos 35(3): 344-382.

Sinha, Sarmila \& Warfa, N. 2013. "Treatment of eating disorders among ethnic minorities in Western settings: A systematic review." Psychiatria Danubina 25(Suppl. 2): 295-299.

Stockford, Clare, Biza Stenfert Kroese, Adam Beesley and Newman Leung (2019). Women's recovery from anorexia nervosa: A systematic review and metasynthesis of qualitative research. Eating Disorders: The Journal of Treatment and Prevention 27(4): 343-368. 
Teo, Thomas 2018. "The consequences of "positivism" in psychology." In Outline of Theoretical Psychology. Palgrave Studies in the Theory and History of Psychology. Palgrave Macmillan, London

Thompson, Becky 1994. A hunger so wide and so deep: American women speak out on eating problems. Minneapolis: University of Minnesota Press.

Wright, Jan \& Valerie Harwood (Eds.) 2009. Biopolitics and the 'obesity epidemic': Governing bodies. New York, NY: Routledge. 